\title{
Critical Points for Random Boolean Networks
}

\author{
James F. Lynch* \\ Department of Mathematics and Computer Science \\ Box 5815 \\ Clarkson University \\ Potsdam, NY 13699-5815
}

\begin{abstract}
A model of cellular metabolism due to S. Kauffman is analyzed. It consists of a network of Boolean gates randomly assembled according to a probability distribution. It is shown that the behavior of the network depends very critically on certain simple algebraic parameters of the distribution. In some cases, the analytic results support conclusions based on simulations of random Boolean networks, but in other cases, they do not.
\end{abstract}

\section{Introduction}

Many dynamical systems are modelled by networks of interacting elements. Examples come from diverse areas of science and engineering and over enormous scales of time and space, from biochemical networks within a cell [1] to food webs [3] and collaboration networks in human organizations [16]. Often, these systems are subjected to random or unpredictable processes. In this article, we are concerned with a class of random networks that S. Kauffman [9, 10] proposed as models of cellular metabolism. These are networks of Boolean gates, where each gate corresponds to a gene or protein, and the network describes the interactions among these chemical compounds. Although Boolean networks capture at least some of the salient features of the operation of the genome, researchers have been mainly interested in certain abstract properties of their dynamics. Kauffman's thesis is that randomly assembled complex systems often exhibit "spontaneous order," that is, even though they are not constructed according to any plan, their behavior is often stable and robust.

Kauffman considered several measures of order, based on the limit cycle that the network enters. Since a Boolean network has a finite number of gates, each of which has two possible states, the network itself has a finite number of states, and it will eventually return to some state it had visited earlier. Since

\footnotetext{
*Research partially supported by NSF Grant CCR-9406809
} 
the network operates deterministically, it will keep repeating this sequence of states, which is called the limit cycle. Among the measures of order that have been considered are

1. The number of stable gates - gates that eventually stop changing state.

2. The number of weak gates - gates that can be perturbed without changing the limit cycle that the network enters.

3. The size of the limit cycle.

The key findings of Kauffman's experiments were that networks constructed from Boolean gates with more than two inputs were usually disordered in all three senses: a significant fraction of the gates never stabilized and, when perturbed, caused the network to enter a different limit cycle, and the size of the limit cycle was exponential in the number of gates. But networks constructed from gates with two inputs tended to be ordered in all three senses, in particular, the average limit cycle size was on the order of the square root of the number of gates.

These results raise many biological and mathematical questions. From the viewpoint of biology, a basic issue is whether these Boolean networks capture the essential features of cellular metabolism. Genes are generally active or inactive, i.e., transcribing their protein or not, and the transition between the two states usually happens on a short time scale. Each gene tends to be directly affected by a small number of proteins. Thus the Boolean network model seems to be at least a rough approximation of cellular metabolic networks. Also, genomes are the result of evolution, which involves random events. However, it would be extremely unlikely that the simple probability distributions used by Kauffman are realistic. He studied two kinds of random networks contructed from 2-input gates. In the first kind, all of the 16 Boolean functions of two arguments are equally likely to be assigned to a gate. This is certainly a reasonable place to start, given the lack of knowledge about the actual distribution of functions in real genomic networks. Two of these 16 functions are constants, i.e., they ignore their inputs and output only one value. Such gates exhibit an extreme form of order, and it seemed possible that their presence was the source of order in networks of 2-input gates. However, Kauffman also ran simulations of randomly constructed networks without constant gates, where the remaining 14 two argument functions were equally likely, and the results were similar to those where all 16 functions were used.

Kauffman proposed another category of functions as the source of order. He called these the canalyzing functions. A canalyzing function is a Boolean function for which there exists some argument and some Boolean value such that the output of the function is determined if the argument has that value. For example, the 2-argument OR function $x_{1} \vee x_{2}$ is canalyzing because if either argument has the value 1 , then the value of $x_{1} \vee x_{2}$ is 1 . Fourteen out of the sixteen 2-argument Boolean functions, including the constant functions, are canalyzing, but this proportion drops rapidly among Boolean functions with 
more than two arguments. Thus the hypothesis that nets with many canalyzing gates tend to be ordered, while those with few of them do not, is consistent with the experimental results.

All of theses definitions and claims have precise mathematical formulations, so a natural question is whether the experimental results are supported by proofs. Interestingly, at about the same time that Kauffman started investigating random Boolean networks, the mathematical techniques for dealing with random networks were being developed by P. Erdős and A. Rényi [5, 6] and E. Gilbert [7], but it was quite some time before any of these techniques were applied to the analysis of random Boolean networks. The first proofs of any of Kauffman's claims appear in an article co-authored by a mathematical biologist (J. Cohen) and a random graph theorist (T. Euczak) [2].

Random graph theory is now a flourishing branch of combinatorics. The most extensively studied version of random graph is the independent edge model. In this version, there is a probability $p$ (which may depend on the number of vertices in the graph) such that for each pair of vertices independently, there is an undirected edge between them with probability $p$. Graph theorists have discovered many deep and interesting results about this kind of random graph, but it does not seem to be a good model of the random networks studied in biology, communications, and engineering. A major distinction is that the degree distribution of this kind of graph is Poisson, but the degree distribution of many real-world networks obeys a power law. A better model for these situations may be random graphs with a specified degree distribution, which are considered in recent articles by M. Molloy and B. Reed [13, 14. Some other shortcomings of the standard version of graph pointed out by M. Newman, S. Strogatz, and D. Watts [15] are that it is undirected and has only one type of vertex. They develop some techniques for dealing with random directed graphs with vertices of several types. However, even this model lacks the structure needed to model the dynamic behavior of networks.

Kauffman's Boolean networks are a further extension of the models in 115 that do include this additional structure. The gates of a Boolean network are vertices assigned a type corresponding to a Boolean function, and the directed edges indicate the inputs to each gate. But instead of simply regarding each vertex as a static entity, we are interested in how the functions of the gates change the state of the network over time. Our random Boolean networks are specified by a sequence of probabilities $p_{1}, p_{2}, \ldots$ whose sum is 1 , where for each gate independently, $p_{i}$ is the probability that it is assigned the $i$ th Boolean function. Once each gate has been assigned its function, its indegree is determined by the number of arguments of the function, and its input gates are chosen at random using the uniform distribution. Lastly, a random initial state is chosen.

Our main results are simple algebraic conditions, derived from the distribution $p_{1}, p_{2}, \ldots$ that imply ordered behavior of the first two kinds mentioned above: almost all gates stabilize quickly, and almost all gates can be perturbed without affecting the long-term behavior of the network. Conversely, if the conditions fail, then the networks do not behave in such an ordered fashion. Our 
conditions actually imply forms of ordered behavior stronger than Kauffman's. That is, the gates stabilize in time on the order of $\log n$, where $n$ is the number of gates, and the effect of a perturbation dies out within order $\log n$ steps. Consequently, the failure of our conditions implies forms of disordered behavior that are weaker than the negations of Kauffman's.

We then apply our main results to the two classes of 2-input Boolean networks mentioned above. Here, our analysis verifies some of Kauffman's claims for networks in the first class, but it casts doubt on similar claims for the other class.

\section{Definitions}

A Boolean network $B$ is a 3 -tuple $\langle V, E, \mathbf{f}\rangle$ where $V$ is a set $\{1, \ldots, n\}$ for some natural number $n, E$ is a set of directed edges on $V$, and $\mathbf{f}=\left(f_{1}, \ldots, f_{n}\right)$ is a sequence of Boolean functions such that for each $v \in V$, the number of arguments of $f_{v}$ is indeg $(v)$, the indegree of $v$ in $E$. The interpretation is that $V$ is a collection of Boolean gates, $E$ describes their interconnections, and $\mathbf{f}$ describes their operation.

The gates update their states synchronously at discrete time steps $0,1, \ldots$. At any time $t$, each gate $v$ is in some state $x_{v} \in\{0,1\}$. Letting $\mathbf{x}=\left(x_{1}, \ldots, x_{n}\right)$, we say that $B$ is in state $\mathbf{x}$ at time $t$. Let indeg $(v)=m$ and $u_{1}<u_{2}<\cdots<u_{m}$ be the gates such that $\left(u_{i}, v\right) \in E$ for $i=1, \ldots, m$. These are referred to as the in-gates of $v$. Then the state of $v$ at time $t+1$ is $y_{v}=f_{v}\left(x_{u_{1}}, \ldots, x_{u_{m}}\right)$. Letting $\mathbf{y}=\left(y_{1}, \ldots, y_{n}\right)$, we put $B(\mathbf{x})=\mathbf{y}$.

The next definitions describe the dynamical properties of Boolean networks that we will analyze.

Definition 1. Let $\mathbf{x} \in\{0,1\}^{n}$.

1. For $t=0,1, \ldots$, we put $B^{t}(\mathbf{x})$ for the state of $B$ at time $t$, given that its state at time 0 is $\mathbf{x}$. That is,

$$
\begin{aligned}
B^{0}(\mathbf{x}) & =\mathbf{x}, \text { and } \\
B^{t+1}(\mathbf{x}) & =B\left(B^{t}(\mathbf{x})\right) \text { for all } t .
\end{aligned}
$$

We also put $B_{v}^{t}(\mathbf{x})$ for $y_{v}$ where $\mathbf{y}=B^{t}(\mathbf{x})$.

2. Gate $v$ stabilizes in $t$ steps on input $\mathbf{x}$ if $B_{v}^{t^{\prime}}(\mathbf{x})=B_{v}^{t}(\mathbf{x})$ for all $t^{\prime} \geq t$.

3. For $\mathbf{x} \in\{0,1\}^{n}$ and $v \in\{1, \ldots, n\}$, we put $\mathbf{x}^{v}$ for the state which is identical to $\mathbf{x}$ except that $x_{v}^{v}=1-x_{v}$.

4. Let $u, v \in\{1, \ldots, n\}$ and $\mathbf{x} \in\{0,1\}^{n}$. We say that $v$ affects $u$ at time $t$ on input $\mathbf{x}$ if $B_{u}^{t}(\mathbf{x}) \neq B_{u}^{t}\left(\mathbf{x}^{v}\right)$. We put $A^{t}(v, \mathbf{x})=\{u \in V$ : $v$ affects $u$ at time $t$ on input $\mathbf{x}\}$.

5. Gate $v$ is t-weak on input $\mathbf{x}$ if $A^{t}(v, \mathbf{x})=\emptyset$, i.e., $B^{t}(\mathbf{x})=B^{t}\left(\mathbf{x}^{v}\right)$. Gate $v$ is $t$-strong on $\mathbf{x}$ if it is not $t$-weak on $\mathbf{x}$. If $\mathbf{x}$ is understood, we simply say $v$ is $t$-weak or $t$-strong. 
For small intervals of time, the dynamical properties described above are determined by the "local" structure of the network. That is, the behavior of a gate over the interval $0,1, \ldots, t$ is determined by the portion of the network consisting of all gates that can reach the gate by a path in $E$ of length at most $t$. Similarly, the gates affected by a given gate lie in the portion consisting of all gates reachable from the gate by such a path. Of course, for large enough $t$, these portions will be the entire network. The next definitions capture these notions of locality.

Definition 2. 1. For any subset $I \subseteq V$,

$$
\begin{aligned}
S_{+}^{0}(I) & =I, \text { and } \\
S_{+}^{t+1}(I) & =\left\{u:(v, u) \in E \text { for some } v \in S_{+}^{t}(I)\right\} \text { for } t \geq 0 .
\end{aligned}
$$

That is, $S_{+}^{t}(I)$ is the set of gates at the ends of paths of length $t$ that start in I. Similarly, $S_{-}^{t}(I)$ is the set of gates at the beginnings of paths of length $t$ that end in $I$.

2. Then

$$
\begin{array}{r}
N_{+}^{t}(I)=\bigcup_{s=0}^{t} S_{+}^{s}(I), \text { and } \\
N_{-}^{t}(I)=\bigcup_{s=0}^{t} S_{-}^{s}(I)
\end{array}
$$

are the out- and in-neighborhoods respectively of I of radius $t$.

We put $S_{+}^{t}(v)$ for $S_{+}^{t}(\{v\})$ and similarly for the other notations. Thus the state of gate $v$ at time $t$ is determined by the states of the gates in $S_{-}^{t}(v)$ and the functions assigned to the gates in $N_{-}^{t-1}(v)$.

As we will show, for sufficiently small $I$ and $t$, the "typical" $N_{+}^{t}(I)$ and $N_{-}^{t}(I)$ induce a forest on $\langle V, E\rangle$, i.e., there are no directed or undirected cycles among their gates. If this is the case for $N_{+}^{t}(v)$, then we can give a simple recursive definition of $A^{t}(v, \mathbf{x})$.

Definition 3. Let $f\left(x_{1}, \ldots, x_{m}\right)$ be a Boolean function of $m$ arguments, and $\mathbf{x}=\left(x_{1}, \ldots, x_{m}\right) \in\{0,1\}^{m}$ be an assignment of 0 's and 1 's to its arguments. For $i \in\{1, \ldots, m\}$, we say that argument $i$ directly affects $f$ on input $\mathbf{x}$ if $f(\mathbf{x}) \neq f\left(\mathbf{x}^{i}\right)$. We extend this notion to gates in a Boolean network in the obvious way. Given a Boolean network $B$ where gate $v$ has in-gates $u_{1}<\cdots<$ $u_{m}$ and state $\mathbf{x} \in\{0,1\}^{n}$, for $i=1, \ldots, m, u_{i}$ directly affects $v$ on input $\mathbf{x}$ if $B_{v}(\mathbf{x}) \neq B_{v}\left(\mathbf{x}^{u_{i}}\right)$.

Lemma 1. Assume $N_{+}^{t}(v)$ induces a tree on $E$. Then for any $s \leq t$, any $\mathbf{x} \in\{0,1\}^{n}$, and any gate $u \in S_{+}^{s}(v), v$ affects $u$ at time $s$ on input $\mathbf{x}$ if and only if 
1. $s=0$ and $u=v$, or

2. $s>0$ and, letting $w$ be the unique gate such that $w \in S_{+}^{s-1}(v) \cap S_{-}^{1}(u)$, $v$ affects $w$ at time $s-1$ on input $\mathbf{x}$, and $w$ directly affects $u$ on input $B^{s-1}(\mathbf{x})$.

\section{Random Boolean Networks}

We will be examining randomly constructed Boolean networks. The random model we use appears to be sufficiently general to capture the particular classes of random Boolean networks in the literature. Let $\phi_{1}, \phi_{2}, \ldots$ be some ordering of all the finite Boolean functions, and let $p_{1}, p_{2}, \ldots$ be a sequence of probabilities such that $\sum_{i=1}^{\infty} p_{i}=1$. The selection of a random Boolean network with $n$ gates is a three stage process. First, each gate is independently assigned a Boolean function using the distribution $p_{1}, p_{2}, \ldots$. That is, for each $v=1, \ldots, n$ and $j=1,2, \ldots$, the probability that gate $v$ is assigned $\phi_{j}$ is $p_{j}$. Next, the ingates for each gate are selected. If the gate has been assigned an $m$-argument function, then its in-gates are chosen from the $\left(\begin{array}{l}n \\ m\end{array}\right)$ equally likely possibilities. Finally, a random initial state is chosen from the $2^{n}$ equally likely possibilities.

We make several restrictions on the distribution $p_{1}, p_{2}, \ldots$ still consistent with the random networks in the literature. Since we are assuming that all orderings of the in-gates to a gate are equally likely, for any $j$ and $k$ such that $\phi_{j}$ and $\phi_{k}$ are identical except for the ordering of their arguments, $p_{j}=p_{k}$. Also, for any $j$ and $k$ such that $\phi_{j}=\neg \phi_{k}, p_{j}=p_{k}$. This implies that, for any gate $v$ such that $N_{-}^{t}(v)$ is acyclic, $B_{v}^{t}(\mathbf{x})$ is equally likely to be 0 or 1 . Lastly, we assume that the average and variance of the number of arguments of a randomly selected Boolean function, or equivalently, the average and variance of the indegree of a gate, are finite. That is, letting each $\phi_{i}$ have $m_{i}$ arguments, $\sum_{i=1}^{\infty} p_{i} m_{i}^{2} \in[0, \infty)$.

\section{Branching Processes}

As will be shown, for $t$ not large compared to $n$, the typical $N_{+}^{t}(v)$ induces a tree in a Boolean network with $n$ gates. A perturbation of the state of such $v$ may cause perturbations to the states of $S_{+}^{1}(v)$ in the next step, then $S_{+}^{2}(v)$, and so on, in a "wave" that propagates through $N_{+}^{t}(v)$. It is possible that this wave dies out and the effects of the perturbation are transient, i.e., gate $v$ is weak. We will show that this behavior can be approximated by a branching process. Then, by applying basic results about branching processes, we will derive our results about weak gates. We will summarize the results that we need. For more information on branching processes, see T. Harris [8].

A branching process can be identified with a rooted labelled tree. The tree may have infinite branches. Each node will be labelled with the unique path from the root to that node. That is, the root is labelled with the null sequence. If the root has $k$ children, they are labelled with the sequences $(1),(2), \ldots,(k)$. If the 
second child of the root has $l$ children, then they are labelled with the sequences $(2,1),(2,2), \ldots,(2, l)$, and so on. Generation $t$ consists of all nodes labelled with a sequence of length $t$. The number of children of any node is independent of the number of children of any other node, but the probability of having a certain number of children is the same for all nodes. Thus the probability space of a branching process is determined by a sequence $\left(q_{k}: k=0,1, \ldots\right)$ where $q_{k}$ is the probability that a node has $k$ children. The probability measure on this space will be denoted by bpr. In describing events in this space, $P$ will denote a branching process. If $\chi$ is a property of branching processes, $P \models \chi$ means $\chi$ holds for $P$, and $\operatorname{bpr}(P \models \chi)$ is the probability that $\chi$ holds.

For $t \geq 0, P\lceil t$ will be the finite labelled tree which is $P$ restricted to its first $t$ generations. $Z_{t}$ will be the random variable which is the size of generation $t$, i.e., the number of nodes of depth $t$.

The generating function of the branching process is the series

$$
F(z)=\sum_{k=0}^{\infty} q_{k} z^{k} .
$$

That is, $F(z)$ is the probability generating function of $Z_{1}$ since $q_{k}=\operatorname{bpr}\left(Z_{1}=\right.$ $k$ ). A basic result is that the $t$-th iterate of $F(z)$ is the probability generating function of $Z_{t}$. The iterates of $F(z)$ are defined by

$$
\begin{aligned}
F_{0}(z) & =z \text { and } \\
F_{t+1}(z) & =F\left(F_{t}(z)\right) \text { for } t \geq 0 .
\end{aligned}
$$

Then

Theorem 1. The probability generating function of $Z_{t}$ is $F_{t}(z)$, i.e.,

$$
F_{t}(z)=\sum_{k=0}^{\infty} \operatorname{bpr}\left(Z_{t}=k\right) z^{k}
$$

This enables us to express the moments of $Z_{t}$ in terms of the moments of $Z_{1}$, which in turn have simple representations in terms of the derivatives of $F(z)$. Let $\mu$ and $\sigma^{2}$ be the first and second moments of $Z_{1}$, that is, $\mu=\mathbf{E}\left(Z_{1}\right)$ and $\sigma^{2}=\operatorname{var}\left(Z_{1}\right)$.

Theorem 2. We have

$$
\begin{aligned}
\mu & =F^{\prime}(1) \text { and } \\
\sigma^{2} & =F^{\prime \prime}(1)+F^{\prime}(1)-\left(F^{\prime}(1)\right)^{2} .
\end{aligned}
$$

More generally, for all $t \geq 0$, the first and second moments of $Z_{t}$ are

$$
\begin{aligned}
\mathbf{E}\left(Z_{t}\right) & =\mu^{t} \text { and } \\
\operatorname{var}\left(Z_{t}\right) & = \begin{cases}\frac{\sigma^{2} \mu^{t}\left(\mu^{t}-1\right)}{\mu^{2}-\mu} & \text { if } \mu \neq 1, \\
t \sigma^{2} & \text { if } \mu=1 .\end{cases}
\end{aligned}
$$




\section{$5 \quad$ Weak Gates}

In this section, $\alpha$ and $\beta$ will be positive constants satisfying $2 \alpha \log \delta+2 \beta<1$ and $\alpha \log \delta<\beta$, where $\delta=\mathbf{E}\left(m_{i}\right)$.

Lemma 2. Let $S \subseteq\{1, \ldots, n\},|S| \leq n^{\beta}$, and $t \leq \alpha \log n$. The following events have probability $1-o(1)$ :

1. For every $v \in S, N_{-}^{t}(v)$ induces a tree in $\langle V, E\rangle$.

2. For every distinct $u, v \in S, N_{-}^{t}(u) \cap N_{-}^{t}(v)=\emptyset$.

Proof. We show that each of these events fails with probability $o(1)$. The calculations are similar for both events, and we show the work only for event 1.

If 1 . fails, then there exist distinct gates $v_{1}, \ldots, v_{s}$ such that

$s \leq \alpha \log n$,

for $i=1, \ldots, s-1, v_{i}$ is an in-gate of $v_{i+1}$, and

$v_{s} \in S$,

and distinct gates $w_{1}, \ldots, w_{r}$ such that

$r \leq \alpha \log n$,

for $i=1, \ldots, r-1, w_{i}$ is an in-gate of $w_{i+1}$,

$w_{1}=v_{1}$, and

for some $h \in\{1, \ldots, s\}, w_{r}=v_{h}$.

Either $h$ above is 1 or greater than 1 . The two cases are similar, and we will describe only the second. Therefore we can assume $r \geq 2$. Now $s, r$, and $h$ can be chosen in $O\left((\log n)^{3}\right)$ ways. The gates $v_{1}, \ldots, v_{s}$ and $w_{2}, \ldots, w_{r-1}$ can be chosen in $O\left(n^{s+r-3+\beta}\right)$ ways. For each $j \in\{1, \ldots, s-1\}-\{h-1\}$, the probability that $v_{j}$ is an in-gate of $v_{j+1}$ is

$$
\begin{aligned}
\sum_{i=1}^{\infty} p_{i} \frac{\left(\begin{array}{c}
n-1 \\
m_{i}-1
\end{array}\right)}{\left(\begin{array}{c}
n \\
m_{i}
\end{array}\right)} & =\sum_{i=1}^{\infty} p_{i} \frac{m_{i}}{n} \\
& =\frac{\delta}{n} .
\end{aligned}
$$

Similarly, the probability that each $w_{j}$ is an in-gate of $w_{j+1}$ for $j=1, \ldots, r-2$ is $\delta / n$. The probability that both $v_{h-1}$ and $w_{r-1}$ are in-gates of $v_{h}$ is

$$
\begin{aligned}
\sum_{i=1}^{\infty} p_{i} \frac{\left(\begin{array}{c}
n-2 \\
m_{i}-2
\end{array}\right)}{\left(\begin{array}{c}
n \\
m_{i}
\end{array}\right)} & =\sum_{i=1}^{\infty} p_{i} \frac{m_{i}\left(m_{i}-1\right)}{n(n-1)} \\
& =O\left(n^{-2}\right) .
\end{aligned}
$$


Altogether, the probability that 1 . fails is

$$
\begin{aligned}
O\left((\log n)^{3} \times n^{s+r-3+\beta} \times(\delta / n)^{s+r-4} \times n^{-2}\right) & =O\left((\log )^{3} \delta^{2 \alpha \log n} n^{\beta-1}\right) \\
& =O\left(\left((\log n)^{3} n^{2 \alpha \log \delta+\beta-1}\right)\right. \\
& =o(1) .
\end{aligned}
$$

We will use the branching process defined as follows. For each $i=1,2, \ldots$ let $\phi_{i}$ have $m_{i}$ arguments, and define

$$
\lambda=\sum_{i=1}^{\infty} p_{i} \sum_{j=1}^{m_{i}} \frac{\mid\left\{\mathbf{x} \in\{0,1\}^{m_{i}}: \text { argument } j \text { directly affects } \phi_{i} \text { on input } \mathbf{x}\right\} \mid}{2^{m_{i}}} .
$$

Thus $\lambda$ may be regarded as the average number of arguments that directly affect a random Boolean function with a random input. Since we are assuming all orderings of the arguments of a Boolean function are equally likely, we can simplify the definition of $\lambda$ to

$$
\lambda=\sum_{i=1}^{\infty} p_{i} m_{i} \frac{\mid\left\{\mathbf{x} \in\{0,1\}^{m_{i}}: \operatorname{argument} 1 \text { directly affects } \phi_{i} \text { on input } \mathbf{x}\right\} \mid}{2^{m_{i}}} .
$$

The branching process is defined by

$$
q_{k}=\frac{\lambda^{k}}{k !} e^{-\lambda}
$$

for $k=0,1, \ldots$ Therefore $F(z)=e^{\lambda z-\lambda}$. From Theorem 2

$$
\begin{aligned}
\mu & =\lambda, \\
\sigma^{2} & =\lambda, \\
\mathbf{E}\left(Z_{t}\right) & =\lambda^{t}, \text { and } \\
\operatorname{var}\left(Z_{t}\right) & = \begin{cases}\frac{\lambda^{t}\left(\lambda^{t}-1\right)}{\lambda-1} & \text { if } \mu \neq 1, \\
t \lambda & \text { if } \mu=1 .\end{cases}
\end{aligned}
$$

Definition 4. Let $T$ be a labelled tree of height $t, B=\langle V, E, \mathbf{f}\rangle$ be a Boolean network, and $\mathbf{x} \in\{0,1\}^{n}$ be its state. For $v \in\{1, \ldots, n\}$, we put $T \Longrightarrow v$ if

$N_{-}^{t}\left(A^{t}(v, \mathbf{x})\right)$ induces a tree in $\langle V, E\rangle$, and

there is an isomorphism from $T$ onto $\left\langle A^{t}(v, \mathbf{x}), E\right\rangle$.

Lemma 3. If $|T| \leq n^{\beta}$ and the height of $T$ is $t \leq \alpha \log n$, then for all $\mathbf{x} \in$ $\{0,1\}^{n}, \operatorname{pr}(T \Longrightarrow v)=\operatorname{bpr}(P\lceil t \cong T)(1+o(1))$. 
Proof. By Lemma 2, if there is an isomorphism $\tau$ from $T$ onto $\left\langle A^{t}(v, \mathbf{x}), E\right\rangle$, then almost surely $N_{-}^{t}\left(A^{t}(v, \mathbf{x})\right)$ induces a tree in $\langle V, E\rangle$. Thus we need only analyze the probability that $\tau$ exists. Let $u_{1}, \ldots, u_{s}$ be the non-leaf nodes of $T$, in lexicographic order. The construction of $\tau$ is recursive and proceeds in stages $1, \ldots, s$. At each stage $j, \tau\left(u_{j}\right)$ has been defined at some previous stage, and it is extended to the children of $u_{j}$. (At stage 1, $\tau\left(u_{1}\right)=v$ has already been defined.) Also, the Boolean functions assigned to these children are selected.

Thus, assume that at stage $j, \tau\left(u_{1}\right), \ldots, \tau\left(u_{K_{j}}\right)$ have already been defined, where $j \leq K_{j}$. Let $u_{j}$ have $k_{j}$ children. Then there are $\left(\begin{array}{c}n-K_{j} \\ k_{j}\end{array}\right)$ ways of selecting the children of $\tau\left(u_{j}\right)$ in $A^{t}(v, \mathbf{x})$. Having chosen these children, we next assign Boolean functions to them. Independently, for each child $w$ of $\tau\left(u_{j}\right)$, let $\phi_{i}$ be assigned to it. This event has probability $p_{i}$, and the probability that $\tau\left(u_{j}\right)$ is an in-gate of $w$ is

$$
\frac{\left(\begin{array}{c}
n-1 \\
m_{i}-1
\end{array}\right)}{\left(\begin{array}{c}
n \\
m_{i}
\end{array}\right)}=\frac{m_{i}}{n} .
$$

Summing over all $i$, we get the probability that $\tau\left(u_{j}\right)$ directly affects $w$ :

$$
\sum_{i=1}^{\infty} \frac{p_{i} m_{i} \times \mid\left\{\mathbf{x} \in\{0,1\}^{m_{i}}: \text { argument } 1 \text { directly affects } \phi_{i} \text { on input } \mathbf{x}\right\} \mid}{n 2^{m_{i}}}=\frac{\lambda}{n} .
$$

Therefore the probability that these $k_{j}$ gates are directly affected by $\tau\left(u_{j}\right)$ is $(\lambda / n)^{k_{j}}$.

Since the events of assigning Boolean functions to all the gates are independent, the probability that the selected gates belong to $A^{t}(v, \mathbf{x})$ is

$$
\begin{aligned}
\prod_{j=1}^{s}\left(\begin{array}{c}
n-K_{j} \\
k_{j}
\end{array}\right)\left(\frac{\lambda}{n}\right)^{k_{j}} & =\left(\prod_{j=1}^{s} \frac{\lambda^{k_{j}}}{k_{j} !}\right)\left(1-\frac{O\left(n^{\beta}\right)}{n}\right)^{O\left(n^{\beta}\right)} \\
& =\left(\prod_{j=1}^{s} \frac{\lambda^{k_{j}}}{k_{j} !}\right)\left(1+O\left(n^{2 \beta-1}\right)\right) .
\end{aligned}
$$

The probability that no other gates are in $A^{t}(v, \mathbf{x})$ is

$$
\left(1-\frac{\lambda s}{n}\right)^{n-|T|}=e^{-\lambda s}\left(1+O\left(n^{2 \beta-1}\right)\right) .
$$

Therefore

$$
\begin{aligned}
\operatorname{pr}(T \Longrightarrow v) & =\left(\prod_{j=1}^{s} \frac{e^{-\lambda} \lambda^{k_{j}}}{k_{j} !}\right)(1+o(1)) \\
& =\operatorname{bpr}(P \nmid t \cong T)(1+o(1)) .
\end{aligned}
$$


We say that a property $\chi$ of branching processes depends only on the first $t$ generations if, for any two branching processes $P_{1}$ and $P_{2}$ such that $P_{1}\lceil t \cong$ $P_{2}\left\lceil t\right.$, either $P_{1} \models \chi$ and $P_{2} \models \chi$, or $P_{1} \not \models \chi$ and $P_{2} \not \models \chi$. Thus $\chi$ can be identified with a set of labelled trees of depth at most $t$. We will also use the notation $\left\langle A^{t}(v, \mathbf{x}), E\right\rangle \models \chi$ to mean $\left\langle A^{t}(v, \mathbf{x}), E\right\rangle$ induces a tree in $\langle V, E\rangle$ whose corresponding branching process satisfies $\chi$.

Theorem 3. Let $\chi$ be a property of branching processes that depends only on the first $\alpha \log n$ generations. Then for all $\mathbf{x} \in\{0,1\}^{n}$

$$
\operatorname{pr}\left(\left\langle A^{t}(v, \mathbf{x}), E\right\rangle \models \chi\right)=\operatorname{bpr}(P \models \chi)+o(1) .
$$

Proof. By the previous lemma, it suffices to show that $\operatorname{bpr}\left(\mid P\left\lceil\alpha \log n \mid \geq n^{\beta}\right)=\right.$ $o(1)$.

If $|P| \alpha \log n \mid \geq n^{\beta}$, then $Z_{t} \geq n^{\beta} /(\alpha \log n)$ for some $t=1, \ldots, \alpha \log n$. Since $\mathbf{E}\left(Z_{t}\right)=\lambda^{t} \leq \delta^{t} \leq n^{\alpha \log \delta}<n^{\beta} /(\alpha \log n$,

$$
\begin{aligned}
& \operatorname{pr}\left(Z_{t} \geq n^{\beta} /(\alpha \log n)\right) \leq \frac{\operatorname{var}\left(Z_{t}\right)}{\left(n^{\beta} /(\alpha \log n)-\mathbf{E}\left(Z_{t}\right)\right)^{2}} \text { by Chebyshev's inequality } \\
& =\left\{\begin{array}{l}
\frac{\lambda^{2 t-1}+\lambda^{2 t-2}+\cdots+\lambda^{t}}{\left(n^{\beta} /(\alpha \log n)-\lambda^{t}\right)^{2}} \text { if } \lambda \neq 1 \\
\frac{t \lambda}{\left(n^{\beta} /(\alpha \log n)-\lambda^{t}\right)^{2}} \text { if } \lambda=1
\end{array}\right. \\
& =o(1 / \log n) \text { in either case. }
\end{aligned}
$$

A gate $v$ such that $N_{-}^{\alpha \log n}\left(A^{\alpha \log n}(v, \mathbf{x})\right)$ is acyclic is $\alpha \log n$-weak if and only if its corresponding branching process is extinct within $\alpha \log n$ generations. Clearly this depends only on the first $\alpha \log n$ generations, so Theorem 3 applies. By basic results from branching process theory, the probability of extinction in $t$ generations is $\operatorname{bpr}\left(Z_{t}=0\right)=F_{t}(0)$, and $\lim _{t \rightarrow \infty} F_{t}(0)=r$, where $r$ is the smallest nonnegative root of $z=F(z)$. Further, when $\mu \leq 1, r=1$, and when $\mu>1, r<1$. Therefore

Theorem 4. There is a constant $r$ such that for all $\mathbf{x} \in\{0,1\}^{n}$

$$
\lim _{n \rightarrow \infty} \operatorname{pr}(v \text { is } \alpha \log n \text {-weak })=r .
$$

When $\lambda \leq 1, r=1$, and when $\lambda>1, r<1$.

Corollary 1. The expected number of $\alpha \log n$-weak gates in a random Boolean network is asymptotic to rn.

A stronger result is

Corollary 2. The number of $\alpha \log n$-weak gates in almost all Boolean networks is asymptotic to $\mathrm{rn}$. 
That is, there is a function $\varepsilon(n)$ such that $\varepsilon(n) \rightarrow 0$ and, letting the random variable $X_{n}$ be the number of $\alpha \log n$-weak gates in a random Boolean network with $n$ gates,

$$
\lim _{n \rightarrow \infty} \operatorname{pr}\left(\left|X_{n}-r n\right| \leq n \varepsilon(n)\right)=1 .
$$

Proof. By the previous corollary,

$$
\mathbf{E}\left(X_{n}\right)=r n+n \varepsilon(n),
$$

where $\varepsilon(n)$ is a function such that $\lim _{n \rightarrow \infty} \varepsilon(n)=0$. When $\lambda \leq 1, r=1$, so, letting the random variable $Y_{n}=n-X_{n}$, by Markov's inequality

$$
\operatorname{pr}\left(Y_{n} \geq n \sqrt{\varepsilon(n)}\right)=O(\sqrt{\varepsilon(n)}) .
$$

Therefore the corollary holds for $\lambda \leq 1$.

When $\lambda>1, r<1$, and we need to estimate $\operatorname{var}\left(X_{n}\right)$. Using methods similar to those in the proofs of Lemma 2 and Theorems 3 and 7 it can be shown that, for any two distinct gates $u$ and $v$, almost surely $N_{-}^{\alpha \log n}\left(A^{\alpha \log n}(u, \mathbf{x})\right)$ and $N_{-}^{\alpha \log n}\left(A^{\alpha \log n}(v, \mathbf{x})\right)$ are acyclic, their intersection is empty, and

$$
\lim _{n \rightarrow \infty} \operatorname{pr}(u \text { and } v \text { are } \alpha \log n \text {-weak })=r^{2} .
$$

Therefore

$$
\operatorname{var}\left(X_{n}\right)=r(1-r) n+n^{2} \varepsilon^{\prime}(n)
$$

for some function $\varepsilon^{\prime}(n) \rightarrow 0$. By Chebyshev's inequality

$$
\begin{aligned}
\operatorname{pr}\left(\left|X_{n}-r n-n \varepsilon(n)\right|>n \sqrt[4]{\varepsilon^{\prime}(n)}\right) & \leq \frac{r(1-r) n+n^{2} \varepsilon^{\prime}(n)}{n^{2} \sqrt{\varepsilon^{\prime}(n)}} \\
& \rightarrow 0
\end{aligned}
$$

and the corollary also holds for $\lambda>1$.

When $\lambda>1$, it is also true that most of the $\alpha \log n$-strong gates affect many other gates when perturbed.

Corollary 3. Let $\lambda>1$. For almost all random Boolean networks, if gate $v$ is $\alpha \log n$-strong, then there is a positive $W$ such that for $t \leq \alpha \log n$, the number of gates affected by $v$ at time $t$ is asymptotic to $W \lambda^{t}$.

Proof. For $t \geq 0$, let $W_{t}=Z_{t} / \mu^{t}\left(=Z_{t} / \lambda^{t}\right.$ in our case). Again by basic results from branching process theory, there is a random variable $W$ such that

$$
\begin{aligned}
\operatorname{bpr}\left(\lim _{t \rightarrow \infty} W_{t}=W\right) & =1 \text { and } \\
\lim _{t \rightarrow \infty} \operatorname{bpr}\left(Z_{t} \neq 0 \text { and } W=0\right) & =0 .
\end{aligned}
$$

From this the corollary follows. 


\section{Forced Gates}

Instead of analyzing the stable gates in a Boolean network, we will study the forced gates. Since a gate stabilizes if it is forced, this is a stronger condition, but it seems to be more amenable to combinatorial analysis.

For the remainder of this section, $t$ will represent a natural number in the range $0, \ldots, \alpha \log n$, and $y$ will be a variable taking on the values 0 and 1 . Given a Boolean function $\phi\left(x_{1}, \ldots, x_{m}\right)$ and $\mathbf{x}=\left(x_{1}, \ldots, x_{m}\right) \in\{0,1, *\}^{m}$, we say that $\mathbf{x}$ forces $\phi$ to $y$ if, for all $\mathbf{x}^{\prime} \in\{0,1\}^{m}$ such that $x_{i}=x_{i}^{\prime}$ whenever $x_{i} \neq *, \phi\left(\mathbf{x}^{\prime}\right)=y$. The $*$ 's are "don't care" values, meaning their value does not affect the value of $\phi$ whenever the remaining arguments agree with $\mathbf{x}$. For example, $\phi$ is forced by every $\mathbf{x} \in\{0,1\}^{m}$; if $\phi$ is a constant function, then it is forced by every $\mathbf{x} \in\{0,1, *\}^{m}$; if $\phi\left(x_{1}, x_{2}\right)=x_{1} \vee x_{2}$, then it is forced to 0 by $(0,0)$ and to 1 by $(0,1),(1,0),(1,1),(1, *)$, and $(*, 1)$. We can now give a recursive definition of forcing for the gates of a Boolean network.

Definition 5. A gate $v$ is forced to $y$ in 0 steps if $f_{v}$ is the constant function $y$.

For $t \geq 0, v$ is forced to $y$ in $t+1$ steps if, letting $u_{1}, \ldots, u_{m}$ be its in-gates, there is $\mathbf{x} \in\{0,1, *\}^{m}$ such that $\mathbf{x}$ forces $f_{v}$ to $y$ and for each $i=1, \ldots, m$ such that $x_{i} \neq *, f_{u_{i}}$ is forced to $x_{i}$ in $t$ steps. We say that $v$ is forced (in some number of steps) if it is forced to 0 or 1.

It is clear that forcing is a stronger condition than stability.

Lemma 4. If a gate in a Boolean network is forced to $y$ in $t$ steps, then it stabilizes to $y$ in $t$ steps.

Further, conditioning on the event that $N_{-}^{t}(v)$ induces a tree, the probabilities that the in-gates of $v$ are forced in $t-1$ steps are independent, and there is a recursive formula for computing the probability that $v$ is forced in $t$ steps. Since $N_{-}^{t}(v)$ is almost surely a tree for the values of $t$ being considered here, the conditional probability given by the recursive formula will be asymptotic to the unconditional probability of being forced in $t$ steps.

For any natural number $m$ and $\mathbf{x} \in\{0,1, *\}^{m}$, let $|\mathbf{x}|_{0}$ be the number of coordinates of $\mathbf{x}$ that are 0 , and similarly for $|\mathbf{x}|_{1}$ and $|\mathbf{x}|_{*}$. For $i=1,2, \ldots$ let $P_{i}^{y}\left(z_{0}, z_{1}\right)$ be the polynomial in $z_{0}$ and $z_{1}$ defined by

$$
P_{i}^{y}\left(z_{0}, z_{1}\right)=\sum_{\substack{\mathbf{x} \in\{0,1, *\}^{m_{i}} \\ \mathbf{x} \text { forces } \phi_{i} \text { to } y}} z_{0}^{|\mathbf{x}|_{0}} z_{1}^{|\mathbf{x}|_{1}}\left(1-z_{0}-z_{1}\right)^{|\mathbf{x}|_{*}} .
$$

Let

$$
G^{y}\left(z_{0}, z_{1}\right)=\sum_{i=1}^{\infty} p_{i} P_{i}^{y}\left(z_{0}, z_{1}\right) .
$$


Recursively, define

$$
\begin{aligned}
G_{1}^{y}\left(z_{0}, z_{1}\right) & =G^{y}\left(z_{0}, z_{1}\right), \text { and for } t \geq 1 \\
G_{t+1}^{y}\left(z_{0}, z_{1}\right) & =G^{y}\left(G_{t}^{0}\left(z_{0}, z_{1}\right), G_{t}^{1}\left(z_{0}, z_{1}\right)\right) .
\end{aligned}
$$

Lemma 5. If $N_{-}^{t}(v)$ induces a tree, then the probability that $v$ is forced to $y$ in $t$ steps is $G_{t+1}^{y}(0,0)$.

From the definition of $G^{y}$ and the symmetry condition $p_{i}=p_{j}$ whenever $\phi_{i}=\neg \phi_{j}$, we have $G^{0}(a, b)=G^{1}(a, b)$ for all $a$ and $b$, and therefore $G_{t}^{0}(0,0)=$ $G_{t}^{1}(0,0)$ for all $t \geq 1$. Therefore letting

$$
G(z)=2 G^{0}(z / 2, z / 2)
$$

and defining

$$
\begin{aligned}
G_{1}(z) & =G(z), \text { and for } t \geq 1 \\
G_{t+1}(z) & =G\left(G_{t}(z)\right)
\end{aligned}
$$

Lemma 6. If $N_{-}^{t}(v)$ induces a tree, then the probability that $v$ is forced in $t$ steps is $G_{t+1}(0)$.

Theorem 5. There exists $g \in[0,1]$ such that

$$
\lim _{n \rightarrow \infty} \operatorname{pr}(v \text { is forced in } \alpha \log n \text { steps })=g .
$$

Further,

$$
\lim _{t \rightarrow \infty} G_{t}(0)=g
$$

and $g$ is a root of the equation

$$
g=G(g)
$$

Proof. Since

$$
\begin{aligned}
P_{i}^{0}(a, b)+P_{i}^{1}(a, b) \leq & \sum_{\mathbf{x} \in\{0,1, *\}^{m_{i}}} a^{|\mathbf{x}|_{0}} b^{|\mathbf{x}|_{1}}(1-a-b)^{|\mathbf{x}|_{*}} \\
& \text { for nonnegative } a \text { and } b \text { such that } a+b \leq 1 \\
& =1, \\
2 G(a) \leq & \sum_{i=1}^{\infty} p_{i} \\
& =1 \text { for } a \leq 1 .
\end{aligned}
$$

This implies that $G(z)$ is a continuous function on $[0,1]$ and all $G_{t}(0)$ are bounded above by 1 . We will show that $G_{t}(0)$ is a strictly increasing sequence in $t$. Then, taking $g=\sup \left(G_{t}(0): t \geq 1\right)$, the theorem follows. 
To show $G_{t}(0)<G_{t+1}(0)$, again assuming that $N_{-}^{\alpha \log n}(v)$ is a tree, note that the event that $v$ is forced to $y$ in $t$ steps is characterized by a collection $\mathcal{C}$ of rooted trees of height at most $t$ whose nodes are labelled with Boolean functions. Each of these trees is contained in the collection $\mathcal{D}$ of rooted labelled trees that characterizes the event that $v$ is forced to $y$ in $t+1$ steps. Further, some of these trees in $\mathcal{C}$ are of height $t$, and their only leaves that are labelled with constant functions have depth $t$. Take any such tree and replace each leaf that is labelled with a constant with a subtree consisting of a node labelled with a nonconstant function and new in-gates all labelled with constants such that the state of the leaf remains unchanged. The new tree belongs to $\mathcal{D}$ but not $\mathcal{C}$ because $v$ will be forced in $t+1$ steps but not $t$ steps. Therefore $\mathcal{D}$ is strictly larger than $\mathcal{C}$, and $G_{t}(0)<G_{t+1}(0)$.

Corollary 4. The expected number of gates that are forced in $\alpha \log n$ steps is asymptotic to gn.

Corollary 5. The number of gates that are forced in $\alpha \log n$ steps in almost all Boolean networks is asymptotic to gn.

\section{Networks of 2-Input Gates}

We now apply the general results of the previous two sections to some networks studied by Kauffman. As mentioned in the Introduction, he suggested that networks with a large proportion of canalyzing gates tend to be stable with high probability. A Boolean function $f\left(x_{1}, \ldots, x_{m}\right)$ is canalyzing if it is forced by some $\mathbf{x} \in\{0,1, *\}^{m}$ where $x_{i} \neq *$ for exactly one $i \in\{1, \ldots, m\}$. Kauffman's claim seems to be supported by experiments indicating that networks constructed from 2-argument Boolean functions usually exhibit stable behavior, while those constructed from Boolean functions with more than 2 arguments do not. Fourteen out of sixteen 2-argument Boolean functions are canalyzing, but this proportion drops rapidly among Boolean functions with more than two arguments. However, our analysis does not support the experimental findings. To explain these results, we classify the 2-argument Boolean functions into three categories.

I. The two constant functions:

$$
f\left(x_{1}, x_{2}\right)=0 \quad \text { and } f\left(x_{1}, x_{2}\right)=1
$$

II. The twelve nonconstant canalyzing functions, consisting of

A. The four functions that depend on one argument:

$$
\begin{aligned}
& f\left(x_{1}, x_{2}\right)=x_{1} \text { and } f\left(x_{1}, x_{2}\right)=\neg x_{1} \\
& f\left(x_{1}, x_{2}\right)=x_{2} \text { and } f\left(x_{1}, x_{2}\right)=\neg x_{2}
\end{aligned}
$$

B. The eight canalyzing functions that depend on both arguments: 


$$
\begin{array}{ll}
x_{1} \vee x_{2} & \text { and } \neg x_{1} \wedge \neg x_{2} \\
\neg x_{1} \vee x_{2} & \text { and } x_{1} \wedge \neg x_{2} \\
x_{1} \vee \neg x_{2} & \text { and } \neg x_{1} \wedge x_{2} \\
\neg x_{1} \vee \neg x_{2} & \text { and } x_{1} \wedge x_{2}
\end{array}
$$

III. The two noncanalyzing functions exculsive or and equivalence:

$$
x_{1} \oplus x_{2} \quad \text { and } x_{1} \equiv x_{2}
$$

Note that each function is paired with its negation. Let $a, b$, and $c$ be the respective sums of the probabilities of the functions of type I, II, and III, i.e., $a$ is the probability that a gate is assigned a function of type I, and so on. We can now express the $\lambda$ parameter of Section 5 (see Equation (2) ) in terms of $a$, $b$, and $c$. Clearly, if $\phi_{i}$ is of type I,

$$
\mid\left\{\mathbf{x} \in\{0,1\}^{2}: \text { argument } 1 \text { directly affects } \phi_{i} \text { on input } \mathbf{x}\right\} \mid=0 .
$$

If $\phi_{i}$ is of type II.A., say $\phi_{i}\left(x_{1}, x_{2}\right)=x_{1}$, then

$$
\mid\left\{\mathbf{x} \in\{0,1\}^{2}: \text { argument } 1 \text { directly affects } \phi_{i} \text { on input } \mathbf{x}\right\} \mid=4,
$$

whereas if $\phi_{i}\left(x_{1}, x_{2}\right)=x_{2}$, then

$$
\mid\left\{\mathbf{x} \in\{0,1\}^{2}: \text { argument } 1 \text { directly affects } \phi_{i} \text { on input } \mathbf{x}\right\} \mid=0 .
$$

If $\phi_{i}$ is of type II.B., say $\phi_{i}\left(x_{1}, x_{2}\right)=x_{1} \vee x_{2}$, then

$$
\mid\left\{\mathbf{x} \in\{0,1\}^{2}: \text { argument } 1 \text { directly affects } \phi_{i} \text { on input } \mathbf{x}\right\} \mid=2 \text {. }
$$

Altogether, the type II functions contribute $b$ to $\lambda$. Lastly, it is easily seen that if $\phi_{i}$ is a type III function, then

$$
\mid\left\{\mathbf{x} \in\{0,1\}^{2}: \text { argument } 1 \text { directly affects } \phi_{i} \text { on input } \mathbf{x}\right\} \mid=4,
$$

and therefore the type III functions contribute $2 c$ to $\lambda$, giving

$$
\lambda=b+2 c .
$$

To analyze the fixed gates, note that $G(z)$ (see Equations (4) and (5)) is a weighted sum of the 16 terms $2 P_{i}^{0}(z / 2, z / 2)$ corresponding to the 2 -argument Boolean functions. This sum can be simplified by using the above classification and pairing of these functions.

If $\phi_{i}$ is the constant function $\phi_{i}\left(x_{1}, x_{2}\right)=0$, then $P_{i}^{0}(z / 2, z / 2)=1$, but if it is the constant function $\phi_{i}\left(x_{1}, x_{2}\right)=1$, then $P_{i}^{0}(z / 2, z / 2)=0$. Therefore the type I functions contribute the term $a$ to $G(z)$.

If $\phi_{i}$ is a type II.A. function, say $\phi_{i}\left(x_{1}, x_{2}\right)=x_{1}$, then $P_{i}^{0}(z / 2, z / 2)=z / 2$. If $\phi_{i}\left(x_{1}, x_{2}\right)=\neg x_{1}$, then $P_{i}^{0}(z / 2, z / 2)=z / 2$ again. If $\phi_{i}\left(x_{1}, x_{2}\right)$ is a type 
II.B. function, say $x_{1} \vee x_{2}$, then $P_{i}^{0}(z / 2, z / 2)=z^{2} / 4$. If it is $\neg x_{1} \wedge \neg x_{2}$, then $P_{i}^{0}(z / 2, z / 2)=z-z^{2} / 4$. Altogether the type II functions contribute the term $b z$ to $G(z)$.

It is easily seen that the two noncanalyzing functions each have $P_{i}^{0}(z / 2, z / 2)=$ $z^{2} / 2$, and therefore $G(z)=a+b z+c z^{2}$. The roots of the equation

$$
z=a+b z+c z^{2}
$$

are 1 and $a / c$. Since $G(z)$ is positive and increasing on $[0,1]$, the smaller of the two roots is also $\lim _{t \rightarrow \infty} G_{t}(0)$. Therefore by Theorem 5 , the probability that a gate is forced in $\alpha \log n$ steps is asymptotic to $\min (1, a / c)$.

In summary, for almost all Boolean networks, almost all gates are $\alpha \log n$ weak if and only if $b+2 c \leq 1$, and almost all gates are forced in $\alpha \log n$ steps if and only if $a / c \geq 1$. Since $a+b+c=1, b+2 c \leq 1$ is equivalent to $c \leq a$. Therefore both types of ordered behavior hold if and only if $a \geq c$.

Kauffman performed extensive simulations on two classes of random networks constructed from 2-argument Boolean functions. In the first class, all 16 of these functions were equally likely to be assigned to a gate. In the second, no constant functions were used, and the remaining 14 functions were equally likely. In the first case, $a=1 / 8, b=3 / 4$, and $c=1 / 8$, giving $\lambda=1$ and $g=1$ as the only solution to Equation (6). Therefore in this case, almost all gates are weak and stable in $\alpha \log n$ steps. But in the second case, $a=0, b=6 / 7$, and $c=1 / 7$, giving $\lambda=8 / 7$ and $g=0$ as the smaller root of (6). Thus in this case, a nontrivial fraction of the gates are $\alpha \log n$-strong and not forced in $\alpha \log n$ steps.

\section{Conclusions and Open Problems}

Our analysis for the case $a \geq c$ supports the experimental results for networks of 2-input gates when all 16 2-argument functions are equally likely. In fact, it gives stronger results than the conclusions of the experiments in three senses. Kauffman's notion of weakness requires only that the network should eventually return to the same limit cycle after a perturbation, but we have shown that with high probability, within $\alpha \log n$ steps, the network will return to exactly the same state it would be in without the perturbation. Also, as mentioned earlier, forcing is a stronger condition than stability. Lastly, the experiments indicated that almost all gates were weak and stabilized for almost all inputs, while we have shown that almost all gates are weak and forced for all inputs.

On the other hand, there is a qualitative difference in the behavior of random Boolean networks when $a<c$, and networks constructed from only the 14 nonconstant 2-argument functions belong to this category. However, this does not necessarily contradict Kauffman's claim that these networks also display ordered behavior since he stated only that, when perturbed they eventually

${ }^{1}$ Articles 111 and 122 contain proofs that $a \geq c$ implies these kinds of ordered behavior; it was conjectured in 12 that they fail when $a<c$. 
return to the same limit cycle, and eventually almost all gates stabilize. It is possible that the effects of a perturbation vanish after $\alpha \log n$ steps, and most gates stabilize after $\alpha \log n$ steps. Thus one open problem is to determine the long-term behavior of nets where $a<c$ (or more generally, when $\lambda>1$ or $g<1$ ), to see if the analysis agrees with the simulations.

We have not addressed the third of Kauffman's notions of order - the size of the limit cycle, which Kauffman claims is of the order $\sqrt{n}$ for 2 -input networks. It has been shown that when $a>c$, not only is the average size of the limit cycle $O(\sqrt{n})$, it is bounded by a constant with probability asymptotic to 1 [1]. However, when $a=c$, the average size of the state cycle is superpolynomial in $n$ [12. To our knowledge, this is the only analytic result that directly contradicts any of Kauffman's claims. The size of the limit cycle is not known when $a<c$. We conjecture that it is superpolynomial in this case also. More generally, it would be interesting to know if the size of the limit cycle is determined by the $\lambda$ or $g$ parameters.

We have shown that one condition, $a \geq c$, imples both a large number of weak gates and a large number of forced gates in networks of 2-input gates. In the general case, two different conditions were used to characterize these forms of order: $\lambda \leq 1$ for weak gates, and $g=1$ for forced gates. Is there a single algebraic condition that characterizes both kinds of order?

Other questions pertain to the effect of increasing the indegree of gates. If we consider networks where each gate has $K$ inputs (using the uniform distribution), then as mentioned in the Introduction, the simulations indicate that when $K=2$, ordered behavior is very likely, but when $K>2$, the networks tend to be disordered. We have described the results for $K=2$ above. A similar analysis for $K>2$ remains to be done. Using a different model of random Boolean network, B. Derrida and Y. Pomeau [4 have provided evidence supporting the simulations. In their version, at each step, each gate is randomly re-assigned its Boolean function and its inputs. They referred to their model as the "annealed" version and Kauffman's as the "quenched" version. They showed that, given any two arbitrary initial states, as the two systems evolved over time, their Hamming distance (the number of gates on which they differ) is approximated by $c_{K} n$ for some constant $c_{K}$ that depends on $K$. When $K=2$, $c_{K}=0$, but when $K>2, c_{K}>0$. Of course, when $K=2$, the quenched model behaves in this way because almost all of the gates are forced. But it is not known whether it holds for quenched models when $K>2$, and the relationship between the annealed and quenched models is not well understood.

Lastly, there is a network model that has some of the properties of both the annealed and quenched models. Here, the gates and their connections are fixed as in the quenched model, but at each step, a random collection of gates updates their states. In other words, the gates operate asynchronously. As with the annealed model, an asynchronous network need not enter a limit cycle, but the other notions of order are still meaningful, and perhaps they can be studied productively. 


\section{References}

[1] J. M. Bower and H. Bolouri, Computational Modeling of Genetic and Biochemical Networks, The MIT Press, Cambridge, MA (2001).

[2] J. E. Cohen and T. Euczak, Stability of veretices in random Boolean cellular automata, Random Structures and Algorithms 2 (1991), 327-334.

[3] J. E. Cohen and C. M. Newman, A stochastic theory of community food webs. I. Models and aggregated data, Proc. R. Soc. London B 224 (1985), 421-448.

[4] B. Derrida and Y. Pomeau, Random networks of automata: a simple annealed approximation, Europhys. Lett. 1 (1986), 45-49.

[5] P. Erdős and A. Rényi, On random graphs, Pub. Math. 6 (1959), 290-297.

[6] P. Erdős and A. Rényi, On the evolution of random graphs, Magyar Tud. Akad. Mat. Kutato Int. Kozl. 5 (1960), 17-61.

[7] E. Gilbert, Random plane networks, J. SIAM 9 (1961), 533-543.

[8] T. E. Harris, The Theory of Branching Processes, Dover Publications, Inc., New York (1989).

[9] S. A. Kauffman, Metabolic stability and epigenesis in randomly connected nets, J. Theoret. Biol. 22 (1969), 437-467.

[10] S. A. Kauffman, The Origins of Order: Self-Organization and Selection in Evolution, Oxford University Press, New York, (1993).

[11] J. F. Lynch, A criterion for stability in random Boolean cellular automata, Ulam Quart. 2 (1993), 32-44.

[12] J. F. Lynch, On the threshold of chaos in random Boolean cellular automata, Random Structures and Algorithms 6 (1995), 239-260.

[13] M. Molloy and B. Reed, A critical point for random graphs with a given degree sequence, Random Structures and Algorithms 6 (1995), 161-179.

[14] M. Molloy and B. Reed, The size of the giant component of a random graph with a given degree sequence, Combinatorics, Probabilitty and Computing 7 (1998), 295-305.

[15] M. E. J. Newman, S. H. Strogatz, and D. J. Watts, Random graphs with arbitrary degree distribution and their applications, Phys. Rev. E 64 (2001), 026118 .

[16] S. Wasserman and K. Faust, Social Network Analysis, Cambridge University Press, Cambridge (1994). 